\title{
Experiments on the Fermi to Tomonaga-Luttinger liquid transition in quasi-1D systems
}

\author{
M. Hilke, ${ }^{1}$ D.C. Tsui, ${ }^{2}$ L.N. Pfeiffer ${ }^{3}$ and K.W. West ${ }^{3}$ \\ ${ }^{1}$ Dpt. of Physics, McGill University, Montréal, Canada H3A 2T8, \\ ${ }^{2}$ Dpt. of Elect. Eng., Princeton University, Princeton, New Jersey, 08544, \\ ${ }^{3}$ Bell Laboratories, Lucent Technologies, Murray Hill, New Jersey, 07974 *
}

(Dated: June 9, 2003)

\begin{abstract}
We present experimental results on the tunneling into the edge of a two dimensional electron gas (2DEG) obtained with GaAs/AlGaAs cleaved edge overgrown structures. The electronic properties of the edge of these systems can be described by a one-dimensional chiral Tomonaga-Luttinger liquid when the filling factor of the 2DEG is very small. Here we focus on the region where the Tomonaga-Luttinger liquid breaks down to form a standard Fermi liquid close to $\nu=1$ and show that we recover a universal curve, which describes all existing data.
\end{abstract}

Keywords: Tomonaga-Luttinger liquid, strongly interacting systems, quantum Hall effect, low dimensional systems, heterostructures, GaAs

In one dimension and in the presence of interactions, a metal can have a Fermi surface in agreement with Luttinger's theorem [1]. However, fermionic quasi-particles are no longer possible and the elementary excitations are replaced by bosonic charge and spin fluctuations dispersing with different velocities. Hence, this onedimensional metal is no longer a Fermi-liquid but a Tomonaga-Luttinger liquid (TLL) 2]. Models describing one-dimensional interacting Fermions were first considered by Tomonaga and Luttinger [3].

While there are a number of systems, which could exhibit $T L L$ behavior, Wen [4] showed that the edge modes of fractional quantum Hall (FQH) states can be described as chiral TLLs. The chirality is due to the presence of a magnetic field, which forces the edge states to propagate in one direction. A unique feature of the chiral $T L L$ is the absence of back-scattering, i.e., no localization can occur, which is in stark contrast to the nonchiral case. For experiments, a key theoretical result is the existence of power-law correlation functions, which lead to the vanishing of the momentum distribution function at $k_{F}$ following a power-law, i.e., $n(k) \sim\left|k-k_{F}\right|^{\alpha}$, where $\alpha$ is related to the interaction strength. As a consequence, the tunneling current-voltage (I-V) characteristics follows $I \sim V^{\alpha}$ and the zero bias conductance follows $\sigma \sim T^{\alpha-1}$. [4] For the particular case, where the filling factor $\nu=1 / 3$, Wen predicted that $\alpha=3$, hence the tunneling current and conductivity should vanish like $I \sim V^{3}$ and $\sigma \sim T^{2}$ respectively. This is very different from the Fermi liquid-to-Fermi liquid tunneling which would be ohmic and independent of temperature.

Following the predictions of Wen [4] and others [5], several experimental attempts were made in order to observe this power-law dependence. The first experiments considered a gate induced constriction to tunnel between two FQH liquids [6, 7]. Unfortunately, in some cases the results were consistent with a power-law [6] but not in others [7]. This was largely attributed to the smoothness of the potential barrier causing the possible reconstruc- tion of the edge and an energy dependent tunneling barrier. Chang et al. [8] avoided this problem by growing a sharp tunneling barrier on the cleaved edge of a two dimensional electron gas (2DEG). They obtained a good power-law over more than a decade in voltage to obtain a tunneling exponent ( $\alpha \simeq 2.7$ at $\nu=1 / 3$ ) close to Wen's prediction.

When moving away from the primary fraction $\nu=1 / 3$ to $\nu=p /(2 n p \pm 1)$ (where $\mathrm{p}$ and $\mathrm{n}$ are positive integers), the edge cannot be described anymore by a single LL edge mode but requires several additional modes, the number and nature of which depends strongly on the particular fraction and, moreover, the disorder becomes important because of possible inter-mode scattering. The overall structure of these states is reviewed in ref. [9]. As a consequence, the recent experimental result from Grayson et al. 10 came as a surprise, because instead of observing a plateau-like structure between $\nu^{-1}=2$ and 3 , as expected from both the composite fermion theory 11, 12 and a disordered edge in the hierarchical model [13], they observed a linear dependence of the exponent on the inverse filling factor, $\alpha \simeq \nu^{-1}$. Recent theories have attempted to account for this behavior using different approaches 14] and are currently under debate.

In this article, we are interested in the transition between the Fermi liquid and the non-Fermi liquid (or TLL) behavior. It is generally expected that at $\nu=1$ the edge should behave as a standard Fermi liquid and all theories agree. Away from $\nu=1$ the situation is very different, for both $\nu<1$ and $\nu>1$. We experimentally probe the edge around this filling factor by using very high mobility 2DEGs (between $1-30 \times 10^{6} \mathrm{~cm}^{-2} / \mathrm{Vs}$ ), which all show well pronounced fractional quantum Hall features. The 2DEG is confined in GaAs/AlGaAs quantum wells. The 2DEG sample is then placed in the molecular beam epitaxy (MBE) chamber and cleaved for a subsequent growth along the (110) direction. First an atomically sharp barrier of $\left(\mathrm{Al}_{x} \mathrm{Ga}_{1-x} \mathrm{As}\right)$ is grown and then a ndoped GaAs layer 15]. We fixed the barrier height to 


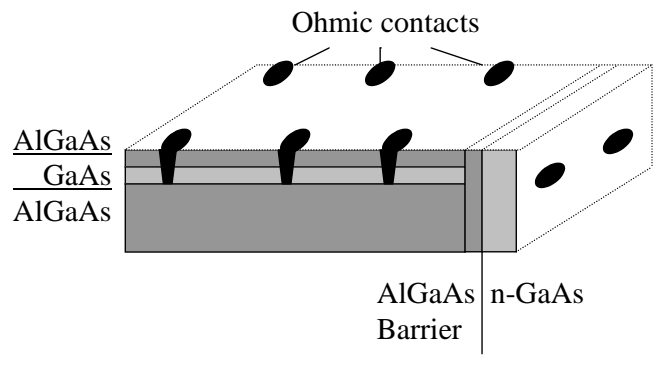

FIG. 1: Sketch of the cleaved edge overgrown structure. The contacts are made by first annealing the Indium 2DEG contacts and then the shallow 3D contacts.

TABLE I: Sample densities.

\begin{tabular}{ccc}
\hline Name & 2D Density & 3D Density \\
\hline PCS2c2 & $1.73 \times 10^{11} \mathrm{~cm}^{-2}$ & \\
PC92a & $3.35 \times 10^{11} \mathrm{~cm}^{-2}$ & $3.25 \times 10^{17} \mathrm{~cm}^{-3}$ \\
PC92c & $3.35 \times 10^{11} \mathrm{~cm}^{-2}$ & $8.5 \times 10^{17} \mathrm{~cm}^{-3}$ \\
PCAN2 & $2.2 \times 10^{11} \mathrm{~cm}^{-2}$ & $4.5 \times 10^{17} \mathrm{~cm}^{-3}$ \\
PCAN4 & $2 \times 10^{11} \mathrm{~cm}^{-2}$ & $4.5 \times 10^{17} \mathrm{~cm}^{-3}$ \\
PCL3 & $1.15 \times 10^{11} \mathrm{~cm}^{-2}$ & \\
\hline
\end{tabular}

about $200 \mathrm{meV}$, by using $x=0.2$ as barrier material. The value of the tunnelling resistance can be tuned by changing the barrier width. We typically used a barrier between $60 \AA$ and $120 \AA$ wide. A sketch of the structure is shown in fig.1.

The n-doped GaAs layer plays a crucial role because it defines the electrostatic potential at the interface between the $2 \mathrm{D}$ and $3 \mathrm{D}$. Indeed, when using a high $3 \mathrm{D}$ doping or density the resistance across the barrier is extremely high $(>G \Omega)$, even at room temperature and independent on the barrier width. We believe that this is due to important electrostatic depletion. In order to analyze the 3D density, we alloyed two shallow ohmic contacts directly on the edge and measured the Shubnikov de Haas oscillations. Typical curves of these are shown in fig. 2. For these densities, the zero field tunnelling resistance depends mainly on the barrier width. A representative list of the different samples is shown in table I below, with their corresponding densities.

In fig. 3 we show a typical four terminal magnetoresistance trace across the barrier. In this particular sample the tunnelling resistance is very low at $\mathrm{B}=0$, which allows us to identify all the quantum Hall features typical of a high mobility 2DEG. Above $6 \mathrm{~T}$ the tunnelling barrier becomes very resistive and we observe an exponential

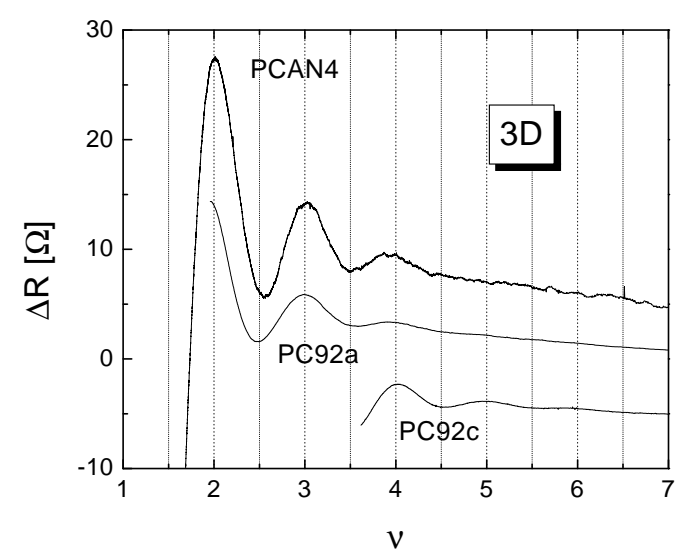

FIG. 2: Magnetoresistance measured directly on the edge as a function of the normalized inverse magnetic field at $40 \mathrm{mK}$. The extracted densities are shown in the table.

increase in tunnelling resistance with $\mathrm{B}$. This is generic to all samples. The main difference between samples is the tunnelling resistance at $B=0$. The overall magnetic field dependence is dominated by an exponential increase of the resistance as a function of $B$, which is due both to the suppression of momentum conservation and to the finite extent of the wave-function given by the magnetic length.

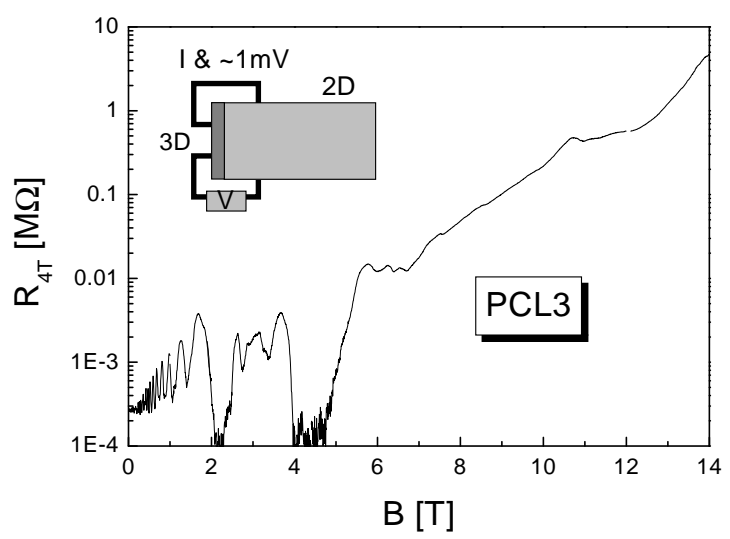

FIG. 3: The magnetoresistance of sample PCL3, measured using a four-terminal configuration, as a function of magnetic field. The data was obtained by applying $1 \mathrm{mV}$ between the $3 \mathrm{D}$ and $2 \mathrm{D}$ and measuring the current with a source-meter. The four-terminal voltage drop is measured by using a voltage amplifier with a high input impedance.

We measured the current-voltage (I-V) characteristics at different magnetic fields. Overall, the I-V's are essentially linear at low fields and become increasingly nonlinear at higher fields. This non-linearity does not de- 
pend on the tunnelling resistance since the behavior is very similar in samples who have very different tunnelling resistances. We found that in all samples the filling factor is the relevant parameter, which determines whether we have a linear or a non-linear I-V. Following the analysis of refs. [8, 10, 16, 17] we extract $\alpha$ from the power-law of the I-V's, using two different methods. We can either use the temperature dependence of the I-V traces or directly the non-linearity in the I-V. A typical temperature dependent $\mathrm{I}-\mathrm{V}$ curve is shown in fig. 4, in which the filling factor is $\nu=2.7$.

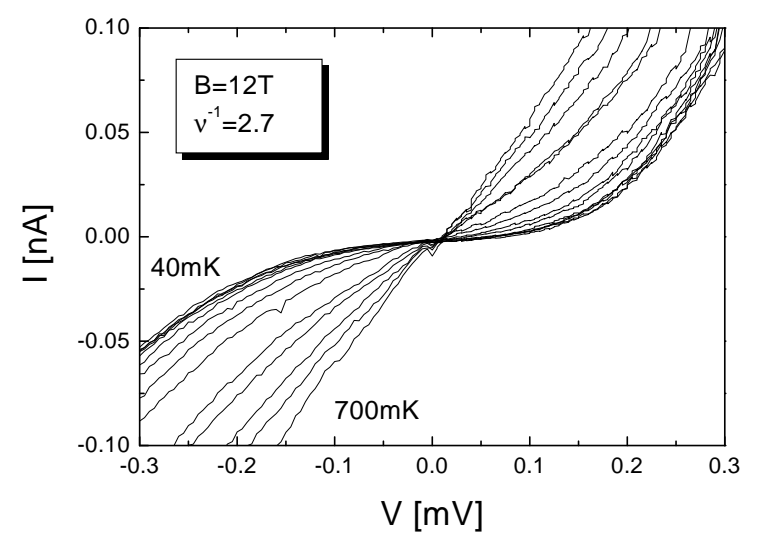

FIG. 4: Temperature dependence of the I-V curves at $12 \mathrm{~T}$, corresponding to a filling factor of $\nu^{-1}=2.7$. This set of curves is taken with sample PCL3.

From the slopes of the $I-V$ traces we can extract the zero bias conductance as a function of temperature, which is plotted in fig. 5 . Above $100 \mathrm{mK}$ the conductance clearly follows a power-law dependence over one decade in temperature. When fitting the data with a power law in that range we obtain a power of $1.64 \pm 0.03$. The saturation below $100 \mathrm{mK}$ is a consequence of the finite input resistance of our voltmeter and not related to the sample. We can avoid this saturation by using a two-terminal configuration for more resistive samples, since in that case the two-terminal contribution from the 2DEG becomes negligible.

In fig. 6 the I-V characteristic is drawn on a log-log scale at base temperature $(40 \mathrm{mK})$. The data follows a power-law over more than a decade in voltage before it starts to deviate significantly from a power-law. We obtain a power of $2.45 \pm 0.01$, when fitting the data below $1 \mathrm{mV}$. Following Wen's [4] argument, this would lead to $\alpha=2.45$. This value is very close to the one obtained from the temperature dependence, i.e., $\alpha=1+1.64$.

We have repeated this procedure for different magnetic fields and different samples. In fig. 7 we have compiled all the values of $\alpha$ as a function of the inverse 2DEG filling factor.

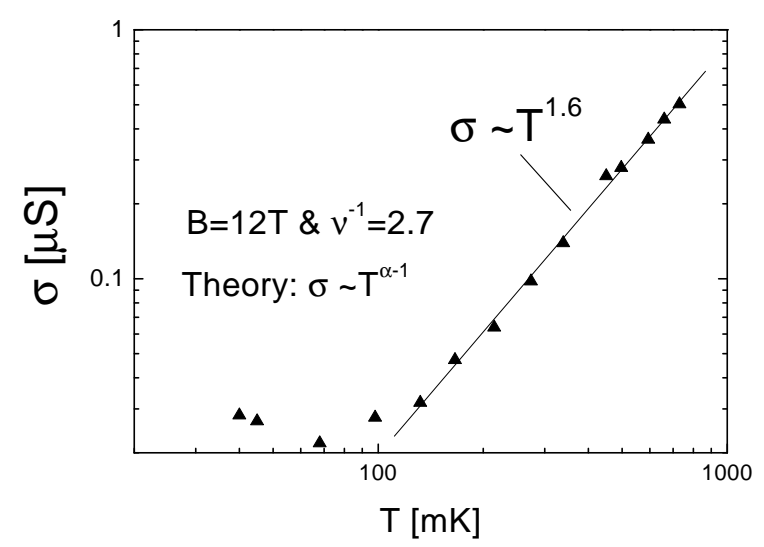

FIG. 5: Temperature dependence of the zero bias conductance at $12 \mathrm{~T}$ obtained from the slopes in fig. 4 .

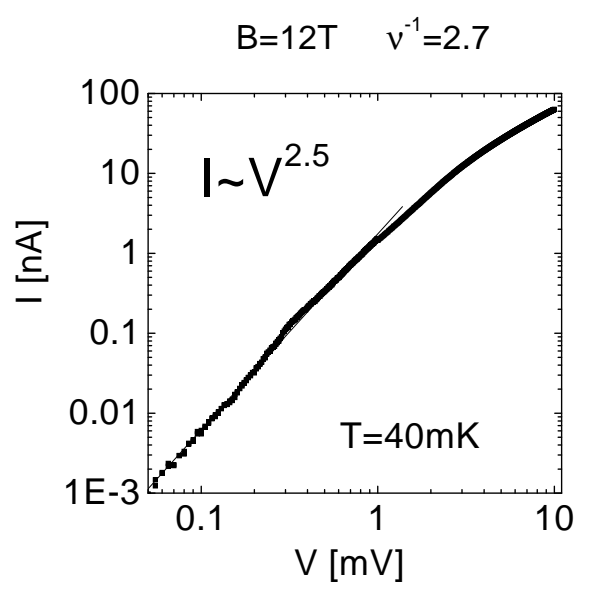

FIG. 6: The I-V characteristics of sample PCL3 at $12 \mathrm{~T}$ and $40 \mathrm{mK}$ on a $\log -\log$ scale.

It is striking that all samples behave qualitatively in a very similar way. Indeed, $\alpha \simeq 1$ for low magnetic fields (low inverse filling factor) and all the way up to a field corresponding to a filling factor between 1 and 1.5. When Increasing the field further there is a transition to a nonlinear regime, in which the exponent increases linearly with the inverse filling factor. The field at which this transition occurs corresponds to the Fermi liquid to TLL transition. [16] Unexpectedly, this transition occurs at a filling factor larger than one, which implies that at $\nu=1$ the system is not a Fermi liquid. In addition, this behavior questions the universal nature of the TLL at the edge of a FQHE system, since $\alpha$ depends on sample parameters.

But; when rescaling the filling factor of all our samples with a value such that $\nu=\alpha=1$ at the linear intercept, 


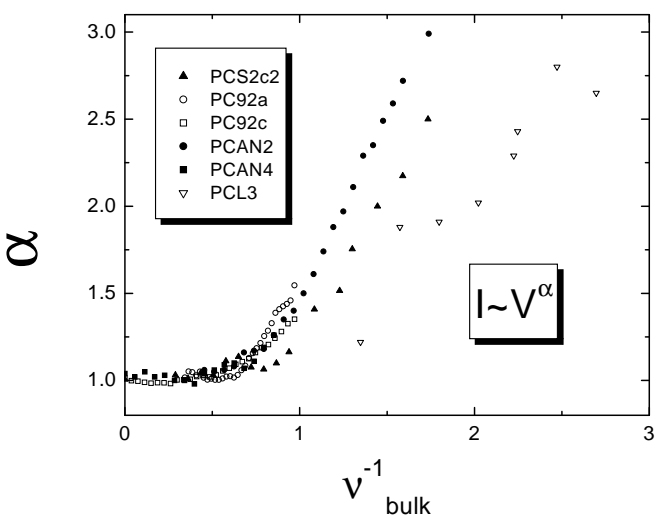

FIG. 7: Exponent $\alpha$ extracted from the power-law part of the $\mathrm{I}-\mathrm{V}$ curves as a function of $B$ and inverse filling factor $\nu^{-1}$ for samples in table I. The errors are within the size of the dots.

we can collapse all our data onto a single curve shown in fig. 8. This rescaling factor depends on the sample growth structure but is identical when using two samples from the same growth. We can perform the same operation on Chang and Grayson's earlier data, [8, 10] by multiplying the filling factor by 1.4. (We haven't included the recent data from ref. 17 since the data cannot be extrapolated consistently all the way down to $\alpha=1$ because most of their data is clustered around $\alpha=3$.) Here again all the data points fall on the same curve. This is very remarkable, considering that the different data points stem from very different sample growth parameters. Indeed, the barrier width varies between $60 \AA$ and $225 \AA$ and the $A l$ content of the barrier between $10 \%$ and $20 \%$. The different $2 \mathrm{D}$ and $3 \mathrm{D}$ densities cover a factor 3 in range.

After rescaling the filling factor, we can describe all the data with the generic form $(\alpha-1)=1.6\left(\nu_{\text {edge }}^{-1}-1\right) \pm 0.2$, when $\nu_{\text {edge }}<1$. We named this rescaled filling factor $\nu_{\text {edge }}$, since the edge filling factor of our 2DEG might be different from the bulk filling factor. The exact dependence of this rescaling factor on sample properties, such as the $2 \mathrm{D}$ and $3 \mathrm{D}$ densities is currently under investigation. It is interesting to note that the extrapolation of our curve to $\alpha=3$ is $\nu^{-1} \simeq 2+1 / 4$, which is slightly off from most existing theories. 4, 5, 9, 11, 12, 13, 14, 19. Moreover, recent theories [12, 19] suggest that the 2DEG density is not monotonic and even oscillates as one approaches the edge. This could be indicative of a complex dependence of the effective edge filling factor $\nu_{\text {edge }}$ on various sample parameters.

In summary, we have presented experimental results on the Tomonaga-Luttinger to Fermi liquid transition at the edge of a 2DEG system close to $\nu=1$. Although the filling factor of the transition is different for different

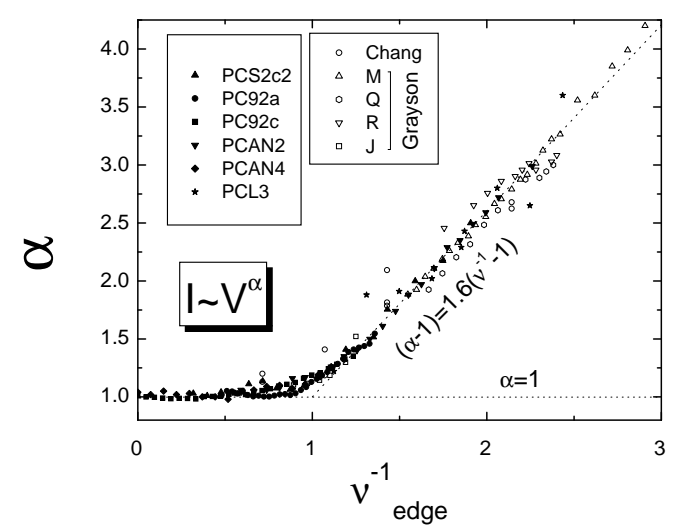

FIG. 8: The chiral TLL exponent $\alpha$ as a function of the scaled inverse filling factor. The scaling factor is chosen in such a way that the linear intercepts (dotted lines) meet at $\nu=\alpha=1$. Our data is presented in filled symbols and earlier data by Chang, Grayson and co-workers [8, 10] is presented in open symbols.

sample structures, we are able to collapse all the data onto a single curve if we rescale the filling factor using a single parameter, hence recovering the notion of a universal behavior in the chiral TLL.

We acknowledge support from NSF, NSERC and FCAR

* hilke@physics.mcgill.ca

[1] J.M. Luttinger, Phys. Rev. 119, 1153 (1960).

[2] F.D.M. Haldane, J. Phys. C 14, 2585 (1981).

[3] J.M. Tomonaga, Prog. Theor. Phys. 5, 544 (1950) and J.M. Luttinger, J. Math. Phys. 4, 1154 (1963).

[4] X.G. Wen Phys. Rev. Lett. 64, 2206 (1990); Phys. Rev. B 41, 12838 (1990); Phys. Rev. B 44, 5708 (1991).

[5] C.L. Kane and M.P.A. Fisher, Phys. Rev. B 46, 2768 (1992); Phys. Rev. B 46, 15233 (1992).

[6] F.P. Milliken, C.P. Umbach and R.A. Webb, Sol. State Comm. 97, 309 (1996); I.J. Maasilta and V.J. Goldman, Phys. Rev. B 55, 4081 (1997); Phys. Rev. B 57, R4273 (1998); P.J. Turley et al., Physica B 249-251, 410 (1998); D.C. Glattli (EP2DS) Physica E (1999).

[7] M. Ando, A. Endo, S. Katsumoto, and Y. Iye, Physica B 249-251, 426 (1998).

[8] A.M. Chang, L.N. Pfeiffer, and K.W. West, Phys. Rev. Lett. 77, 2538 (1996).

[9] X.G. Wen, Adv. Phys. 44, 405 (1995).

[10] M. Grayson, D.C. Tsui, L.N. Pfeiffer, K.W. West, and A.M. Chang Phys. Rev. Lett. 80, 1062 (1998).

[11] A.V. Shytov, L.S. Levitov and B.I. Halperin Phys. Rev. Lett. 80, 141 (1998).

[12] L.S. Levitov, A.V. Shytov and B.I. Halperin, cond-mat/0005016

[13] C.L. Kane, M.P.A. Fisher and Polchinski Phys. Rev. Lett. 
72, 4129 (1994).

[14] S. Conti and G. Vignale, J. Phys. - Cond. Mat.. 10, L779 (1998); J.H. Han and D.J. Thouless, Phys. Rev. $B$ 55, 1926 (1997);J. H. Han, Phys. Rev. B 56, 15806 (1997); U. Zülicke and A.H. MacDonald, Phys. Rev. B 60, 1836 (1999); A.M.M. Pruisken, B. Skoric and M.A. Baranov, Phys. Rev. B 60, 16838 (1999); A. Alekseev, V. Cheianov, A.P. Dmiriev, V. Yu Kachorovskii, cond-mat/9904076 A. Lopez and E. Fradkin, Phys. Rev. B 59, 15323 (1999); D.V. Khveshchenko, Sol. St. Comm. 111, 501 (1999); D.-H. Lee and X.-G. Wen, cond-mat/9809160

[15] L.N. Pfeiffer, K.W. West, H.L. Stormer, J.P. eisentstein, K.W. Baldwin, D. Gershoni and J. Spector, Appl. Phys.
Lett. 56, 1697 (1990).

[16] M. Hilke, D. C. Tsui, M. Grayson, L. N. Pfeiffer, and K. W. West, Phys. Rev. Lett. 87, (2001).

[17] A.M. Chang, M.K. Wu, C.C. Chi, L.N. Pfeiffer and K.W. West, Phys. Rev. Lett. 86, 143 (2001).

[18] M. Grayson, D.C. Tsui, L.N. Pfeiffer, K.W. West and A.M. Chang, Phys. Rev. Lett. 86, 2645 (2001).

[19] V.J. Goldman and E.V. Tsiper, Phys. Rev. Lett. 86, 5841 (2001), S.S. Mandal and J.K. Jain, Sol. State Com. 118,503 (2001), X. Wan, K. Yang, and E.H. Rezayi, Phys. Rev. Lett. 88, 056802 (2002) and S. S. Mandal and J. K. Jain, Phys. Rev. Lett. 89, 096801 (2002). 